\title{
Die praktische Anwendung eines Zytokinprofiles für Diagnose und Verlaufskontrolle bei systemischer juveniler idiopathischer Arthritis: 3 Fallberichte
}

Die systemische juvenile idiopathische Arthritis ( $s \mid \mathrm{IA}$ ) ist eine multifaktorielle autoinflammatorische Erkrankung, die durch Inhibition von Interleukin-1 (IL-1) und Interleukin-6 (IL-6) häufig zur Remission gebracht werden kann.

Durch Nutzung des „Window of Opportunity“ zum Beginn der biologischen Therapie kann die Erkrankung oft in der initialen Phase der Aktivität der „Innate Immunity“ gestoppt werden, bevor die Stimulation der „Adaptive Immunity“ mit Bildung von autoreaktiven T-Zellen und Autoantikörpern auftritt, die bei bis zu $30 \%$ der Patienten trotz zeitgemäßer Therapie zu einer therapieresistenten destruktiven Polyarthritis führen kann [1, 2].

Unter erfolgreicher IL-1- oder IL-6-Blockade bei Patienten mit sJIA werden Akute-Phase-Zeichen wie BSG, CRP, Ferritin, Se-Amyloid völlig blockiert, sodass die Beurteilung der aktuellen Entzündungsaktivität zur Therapiesteuerung durch Messung dieser Parameter schwierig ist. Die überschießende Aktivierung des Immunsystems bei sllA erkennt man daran, dass aktivierte Phagozyten große Mengen der S100-Proteine Calprotectin und S100A12 sowie der proinflammatorischen Zytokine IL-1 $\beta$, IL-6 und IL-18 freisetzen [13].

Untersuchungen der Serumspiegel von S100A12 und Calprotectin wurden als diagnostischer Parameter zur Unterscheidung von s|IA von anderen fieberhaften entzündlichen Erkrankungen beschrieben [14]. Extrem erhöhte Serumspiegel von IL-18 wurden als diagnostischer Marker für sJIA und Morbus Still (aoSD) bei Erwachsenen beschrieben [15].

Wir hatten die Möglichkeit, ein „Zytokinprofil“ bestehend aus Calprotectin, S100A12, IL-1 $\beta$, IL-6, TNF- $\alpha$, IL-18, sIL2-R und Interferon- $y$ im Serum zu bestimmen. Bei drei Patienten der Kinderrheumaambulanz des SMZO Wien war die Bestimmung dieses Zytokinprofiles hilfreich zur Sicherung bzw. zum Ausschluss der Diagnose sJIA, als Verlaufsparameter unter der The-

- Tab. 1 Zytokinprofil, Kits

\begin{tabular}{|l|l|}
\hline Zytokine & Kits \\
\hline SIL2R & Quantikine Elisa, R \&D Systems \\
\hline IL-18 & Human IL-18 Elisa Kit, MBL \\
\hline S100A12 & Human S100A12 Elisa, BioVendor \\
\hline S100A8/9, Calprotectin & IDK Calprotectin Elisa, Immundiagnostik AG \\
\hline IL-6 & IL-6 EASIA, BioSource Europe S. A. \\
\hline TNF- $\alpha$ & TNF- $\alpha$ Elisa, DIAsource Immunoassays S. A. \\
\hline IL-1 & IL-1 $\beta$ EASIA, BioSource Europe S. A. \\
\hline
\end{tabular}

rapie sowie vor und nach Beendigung der Biologikatherapie.

\section{Fallbericht 1}

Das vorher gesunde 8-jährige Mädchen erkrankte im August 2018 während eines Auslandsaufenthaltes an persistierendem Fieber $>40^{\circ} \mathrm{C}$, Exanthem an Oberschenkeln und Oberarmen, Arthralgien in groBen Gelenken und mit sehr hohen Entzündungszeichen (CRP 277,8 mg/l, Ferritin $2285 \mu \mathrm{g} / \mathrm{l}$ ). Es wurde die Diagnose sJIA gestellt und eine Therapie mit Prednisolon mit $1 \mathrm{mg} / \mathrm{kg} / \mathrm{d}$ und Anakinra mit $2 \mathrm{mg} / \mathrm{kg} / \mathrm{d}$ s.c. begonnen. Nach 2 Wochen wurde die Patientin zur weiteren Betreuung in unserer Kinderrheumaambulanz in Wien vorgestellt. Zu diesem Zeitpunkt war sie unter der Therapie in klinischer Remission.

Die laborchemischen Entzündungszeichen waren negativ: BB normal, CRP negativ, Se-Amyloid 11,7 mg/l, Ferritin $242 \mu \mathrm{g} / \mathrm{l}$, LDH 210 U/I. Die Bestätigung der Diagnose zur Beurteilung der Frage nach der Fortführung der Therapie war daher notwendig. Im Zytokinprofil zeigten sich 2 Wochen nach Beginn der Therapie ein mäßiggradig erhöhtes Calprotectin, ein signifikant erhöhtes IL-18 und ein grenzwertiger sIL2R. IL-1 $\beta$ war mäßiggradig erhöht ( $\triangleright$ Tab. 2). Wir sahen darin die Bestätigung der Diagnose sJIA, setzten die Therapie mit Anakinra unverändert fort und schlichen Prednisolon über 10 Tage aus. Bei den folgenden Kontrollen war die Patientin beschwerdefrei, alle laborchemischen Entzündungsparameter waren negativ. Nach 3 Monaten war das
Zytokinprofil eindeutig negativ, wir beendeten die Therapie mit Anakinra nach insgesamt 4 Monaten [1].

Auch nach einem weiteren Monat waren keine klinischen und laborchemischen Entzündungszeichen nachweisbar, das Zytokinprofil zeigte keine Aktivität an.

\section{Fallbericht 2}

Der jetzt 12-jährige Knabe erkrankte im Juni 2016 an einer Arthritis des linken Kniegelenkes und des PIP-Gelenkes II rechts sowie mit subfebrilen Temperaturen. Bei der auswärtigen stationären Aufnahme im August 2016 zeigten sich sehr hohe Entzündungszeichen: CRP max. 105,9 mg/l, BSC 105/116. Desweiteren fanden sich folgende Befunde: LDH max. 283 U/I, Ferritin max. $415 \mathrm{ng} / \mathrm{ml}$, ANA positiv, CCP-AK schwach positiv, Rheumafaktor negativ, HLA-B27 negativ. Es bestand darüber hinaus keine auffällige Familienanamnese für eine entzündlich rheumatische Erkrankung, eine Psoriasis oder eine chronisch entzündliche Darmerkrankung.

Unter antibiotischer und symptomatischer Therapie kam es zu Fieber bis $40^{\circ} \mathrm{C}$ für 2 Tage und einem flüchtigen Exanthem. Es wurde die Diagnose sJIA gestellt und eine Therapie mit Naproxen und oralen Steroiden für 2 Monate mit sehr gutem Ansprechen durchgeführt. Nach Absetzen der Steroide kam es 10/2016 zu Arthritiden im linken Knie, im linken Ellbogen und im PIP III rechts sowie zu Fieber für 2 Tage. Die Therapie wurde um Canakinumab erweitert, 
- Tab. 2 Zytokinprofil Patientin 1

\begin{tabular}{|c|c|c|c|c|c|c|c|c|}
\hline & Calprotectin & S100A12 & IL-18 & Interferon- $\gamma$ & SIL2R & IL-6 & TNF- $\alpha$ & IL-1 $\beta$ \\
\hline normal & $<4137 \mathrm{ng} / \mathrm{ml}$ & $19-157 \mathrm{ng} / \mathrm{ml}$ & $106-319 \mathrm{pg} / \mathrm{ml}$ & $<0,5 \mathrm{IU} / \mathrm{ml}$ & $210-1179 \mathrm{pg} / \mathrm{ml}$ & $<20 \mathrm{pg} / \mathrm{ml}$ & $<20 \mathrm{pg} / \mathrm{ml}$ & $<10 \mathrm{pg} / \mathrm{ml}$ \\
\hline 09/2018 & 10093 & 140 & 3652 & neg & 1318 & 8,2 & 10,4 & 63,1 \\
\hline $11 / 2018$ & 1495 & 36 & 262 & neg & 1251 & 7,3 & 69 & $<6,5$ \\
\hline $01 / 2019$ & 2543 & 38 & 266,7 & $<0,4$ & 743 & $<7,3$ & 20,7 & $<6,6$ \\
\hline
\end{tabular}

- Tab. 3 Zytokinprofil Patient 2

\begin{tabular}{|c|c|c|c|c|c|c|c|}
\hline & Calprotectin & S100A12 & IL-18 & sIL2R & IL-6 & TNF-alpha & IL-1ß \\
\hline normal & $<4137 \mathrm{ng} / \mathrm{ml}$ & 19-157 ng/ml & $106-319 \mathrm{pg} / \mathrm{ml}$ & $210-1179 \mathrm{pg} / \mathrm{ml}$ & $<20 \mathrm{pg} / \mathrm{ml}$ & $<20 \mathrm{pg} / \mathrm{ml}$ & $<10 \mathrm{pg} / \mathrm{ml}$ \\
\hline $10 / 2016$ & nicht gemessen & nicht gemessen & 453,1 & 1721 & 71,3 & 19,4 & $<6,6$ \\
\hline $08 / 2018$ & 23354 & 1076 & 315 & 1759 & 100,2 & 26,4 & 16,1 \\
\hline $03 / 2019$ & 20158 & 592 & 363,9 & 1654 & 50,1 & 83,1 & $<6,6$ \\
\hline
\end{tabular}

die Steroide fortgesetzt und ab April 2017 zusätzlich Methotrexat verabreicht. Letzteres wurde wegen fehlender Wirkung im Dezember 2017 wieder beendet.

Im Juli 2018 verschlechterte sich die Gelenksituation erheblich. Im August 2018 stellte sich der Patient dann in unserer Ambulanz vor. Zu diesem Zeitpunkt wurde er mit Canakinumab in 6-wöchentlichen Abständen und $5 \mathrm{mg}$ Prednisolon täglich oral behandelt.

Er wies ausgeprägte Entzündungen mit asymmetrischem Befall von 7 vorwiegend großen Gelenken mit Aussparung der Hüften, Handgelenke und der Wirbelsäule auf. Es zeigten sich mäßig erhöhte Entzündungszeichen ohne klinische Systemmanifestationen: BSG 21/41, CRP 24,3 mg/l, Se-Amyloid 61,8 mg/l. Weitere Laborbefunde umfassten: Ferritin 23 $\mu \mathrm{g} / \mathrm{l}, \mathrm{LDH} 242 \mathrm{U} / \mathrm{l}$, ANA positiv mit homogenem Muster, ENA negativ, Rheumafaktoren negativ.

Im Zytokinprofil vom August 2018 zeigten sich ein stark erhöhtes Calprotectin und S100A12. IL-18, sIL2-R sowie IL-1 $\beta$ waren nicht signifikant erhöht, IL-6 deutlich erhöht. Retrospektiv zeigten sich ähnliche Werte aus vorhandenem tiefgefrorenem Serum von 10/2016 ( Tab.3).
Wir führten intraartikuläre Injektionen von Triamcinolon-Hexacetonid in Knie- und Sprunggelenke durch und beendeten die Canakinumab-Therapie. Passager wurden niedrig dosiert orale Steroide und Naproxen verabreicht, die fehlenden Impfungen ergänzt und die medikamentöse Therapie ab Oktober 2018 mit Methotrexat und Etanercept fortgeführt. Der Patient zeigte zunächst ein sehr gutes Ansprechen der Arthritis auf die neue Therapie.

Leider kam es im November 2018 zu einer einseitigen komplizierten Uveitis links mit Reduktion des Sehvermögens des Auges auf $5 \%$. Der Patient wurde mit einer Augenlokaltherapie, einem i.v.-Methylprednisolonpuls für 3 Tage und oralen Steroiden behandelt. Die biologische Therapie wurde auf Adalimumab gewechselt, da unter Etanercept Durchbrüche von Uveitis bei Patienten mit JIA beschrieben sind [11]. Darunter bildeten sich die lokalen Entzündungszeichen im Auge innerhalb von 6 Wochen völlig zurück, allerdings verblieben Synechien des linken Auges. Nach 3 Monaten Therapie mit Methotrexat und Adalimumab hatte sich das Sehvermögen auf 70\% gebessert.

Im März 2019 kam es wieder zu einer deutlichen Arthritis beider Kniegelenke ohne systemische Manifestationen begleitet von deutlich erhöhten Serumkonzentrationen von Calprotectin, S100A12 sowie erhöhtem
IL-6. Die Behandlung erfolgte erneut mit intraartikulären Injektionen von TriamcinoIon-Hexacetonid.

\section{Fallbericht 3}

Das vorher gesunde 7 Jahre alte Mädchen kam im Juli 2017 mit zunehmendem Fieber mit Anstiegen bis $40^{\circ} \mathrm{C} 3$-mal täglich, passagerem urtikariellem Exanthem, starkem Schwitzen, flüchtiger Arthritis der Knie- und Sprunggelenke sowie des linken Handgelenkes und geringer Splenomegalie zur Aufnahme.

Folgende Laborwerte wurden ermittelt: CRP max. 49,8 mg/l, Se-Amyloid max. $175 \mathrm{mg} / \mathrm{l}$, BSG max. 72/97, LDH max. $771 \mathrm{U} / \mathrm{l}$, Ferritin max. $1348 \mathrm{ng} / \mathrm{ml}$, Thrombozyten min. 176 G/I, GOT max. $71 \mathrm{U} / \mathrm{l}$, Triglyzeride $115 \mathrm{mg} / \mathrm{dl}$, Autoantikörper negativ. Im Zytokinprofil zeigten sich exzessiv erhöhte Werte für Calprotectin, S100A12, IL-18, sIL2R sowie erhöhte Werte für IL-6 und Interferon-y ( Tab.4). Anhalt für eine maligne Erkrankung bestand nicht.

Bei den genetischen Untersuchungen zeigten sich insgesamt 5 heterozygote Mutationen (RFX5, TYK2, CCR6, DOCK8, UNC13D) von Immundefekten mit autosomal rezessivem Erbgang. UNC13D-Mutationen in homozygoter Form sind eine Ursache für eine familiäre hämophagozytische Lymphohistiozytose [6]. 
- Tab. 4 Zytokinprofil Patientin 3

\begin{tabular}{|l|l|l|l|l|l|l|}
\hline & Calprotectin & S100A12 & IL-18 & Interferon- $\mathbf{~}$ & slL2R & IL-6 \\
\hline normal & $<4137 \mathrm{ng} / \mathrm{ml}$ & $19-157 \mathrm{ng} / \mathrm{ml}$ & $106-319 \mathrm{pg} / \mathrm{ml}$ & $<0,5$ U U/ml & $210-1179 \mathrm{pg} / \mathrm{ml}$ & $<20 \mathrm{pg} / \mathrm{ml}$ \\
\hline $07 / 2017$ & 46914 & 847 & 201309 & 3 & 17656 & 83,4 \\
\hline $12 / 2017$ & 16468 & 318 & 1741,7 & nicht gemessen & 2487 & 37,1 \\
\hline $06 / 2018$ & 17625 & 307 & 1039,7 & nicht gemessen & 1312 & 96,7 \\
\hline $12 / 2018$ & 4297 & 74 & 692,2 & nicht gemessen & nicht gemessen & nicht gemessen \\
\hline
\end{tabular}

Die EULAR-Kriterien 2016 für ein sekundäres Makrophagen-Aktivierungs-Syndrom bei sJIA (siehe Kasten) waren laborchemisch zwar erfüllt, wegen des guten Allgemeinzustandes und der fehlenden MAS-typischen Symptome wurde die Diagnose zunächst nicht weiter verfolgt.

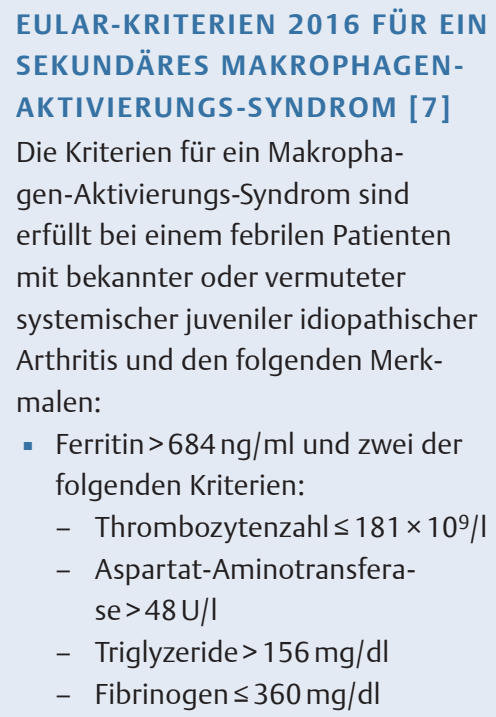

3 Wochen nach Beginn der Symptome entfieberte die Patientin unter symptomatischer Therapie. Im Verlauf von weiteren 1 1⁄2 Jahren blieb sie klinisch gesund, es zeigten sich laborchemisch keinerlei Entzündungszeichen und ein langsames Abfallen von Calprotectin, S100 A12, sIL2R und IL-18 [3].

\section{Diskussion}

Die Diagnose einer sJIA ist in der Initialphase oft schwierig zu sichern. Schwerkranke Kinder mit persistierendem Fieber mit hohen Entzündungszeichen haben eine lange Reihe von Differenzialdiagnosen, die Arthritis tritt oft erst nach Monaten auf und das Exanthem ist häufig unspezifisch und sehr flüchtig, Hepatosplenomegalie und Lymphadenitis sind oft nicht signifikant. Die bisher verwendeten Laboruntersuchungen sind höchst unspezifisch (CRP, Ferritin, LDH, Se-Amyloid, Leukozytose usw.) und bei einer Reihe von Infektionen, Autoimmun- und autoinflammatorischen Erkrankungen, malignen Erkrankungen ähnlich verändert.

Die in unserem Zytokinprofil verwendeten Parameter sind 2 S100-Proteine (Calprotectin und S100A12), 5 proinflammatorische Zytokine (IL-1, IL-6, IL-18, TNF- $\alpha$, Interferon- $\gamma$ ) sowie der sIL2-Rezeptor- $\alpha$.

\section{S100-Proteine}

Die S100-Proteine werden als Alarmproteine aus Zellen der Innate Immunity freigesetzt und stimulieren über Toll-like-Rezeptoren das Inflammasom, das letztlich IL-1 $\beta$ freisetzt, und über IL-6 die klassische Akute-Phase-Reaktion mit Fieber, CRP, Leukozytose usw. hervorruft. Durch medikamentöse IL-1- und IL-6-Blockade wird die Entzündungsinformation nicht an die folgenden Zellen weitergegeben, die messbaren Akute-Phase-Parameter bilden sich daher völlig zurück. Die S100-Proteine lassen daher einen Blick auf die aktuelle Aktivität der Zellen der Innate Immunology am Ort der Inflammation zu.

\section{Interleukin-18}

Interleukin-18 (IL-18) ist ein proinflammatorisches Zytokin aus der Interleukin1-Familie und wird von aktivierten Makrophagen produziert. Es triggert die Th1-Antwort und induziert die Sekretion von Interferon-y durch zytotoxische CD8und NK-Zellen sowie die hämophagozytische Transformation von Makrophagen. Hohe Spiegel von IL-18 treten im Serum, in Synovialbiopsien, Lymphknoten und in der Leber von Patienten mit AoSD und sJIA auf.
Die Bioaktivität von IL-18 wird von seinem Binding Protein, IL18BP, geregelt.

Hohe Spiegel von IL-18 können eine sJIA mit hoher Sensitivität und Spezifität von der Kontrollgruppe sowie von Sepsis, ALL und artikulärer JIA differenzieren [4]. Leek et al . [5] geben den Cut-off von IL-18 für die Diagnose des juvenilen Morbus Still mit $1500 \mathrm{pg} / \mathrm{ml}$ im Serum an.

Feist et al [3] geben an, dass bei Patienten in Remission die IL-18-Werte unter 1000 $\mathrm{pg} / \mathrm{ml}$ sinken und in nachhaltiger Remission die Normalwerte erreichen. Patienten, bei denen während der Ausschleichphase der Steroide die IL-18-Spiegel > 1000 pg/ $\mathrm{ml}$ verblieben, hatten innerhalb eines Jahres häufiger Relapse. Der Cut-off für die Entwicklung eines MAS wird mit 52000 pg/ml angegeben.

\section{Interleukin-6}

Interleukin-6 (IL-6) spielt eine wichtige Rolle in der Pathogenese der sJIA, erhöhte Spiegel im Serum und in der Synovialflüssigkeit korrelieren mit der Schwere der Gelenkserkrankung. Xia et al [4] geben an, dass IL-6-Spiegel bei sJIA höher waren als in der Kontrollgruppe, aber keine signifikanten Unterschiede zur Sepsis bzw. Morbus Kawasaki zeigten und daher nicht als Biomarker zur Differenzialdiagnose der sJIA geeignet sind.

\section{Löslicher Interleukin-2-Rezeptor- $\alpha$}

Der Iösliche Interleukin-2-Rezeptor- $\alpha$ (sIL2-R) ist ein Marker für die T-Zell-Aktivierung und wird von aktivierten B-Zellen, T-Zellen und malignen Zellen freigesetzt. Er ist erhöht bei malignen Erkrankungen, Infektionen, Autoimmunerkrankungen und bei autoimmun lymphoproliferativem Syndrom. Die HLH-2004 Guidelines [9] geben eine hohe Sensitivität und Spezifität bei 
Werten > 2400 U/ml für Makrophagen-Aktivierungs-Syndrom an. Bei sIL2-R> $10000 \mathrm{U} /$ $\mathrm{ml}$ wird eine schlechte Prognose bei HLH angegeben [8].

\section{Interferon-gamma}

Interferon-gamma (IFN-y) wird von T-Lymphozyten und NK-Zellen gebildet und aktiviert zytotoxische T-Zellen und Makrophagen. Es spielt eine zentrale Rolle in der Pathogenese der Makrophagenaktivierung und verläuft parallel zu sIL2-R [8].

Bei unseren Patienten war die Bestimmung des Zytokinprofiles zur Sicherung bzw. zum Ausschluss der Diagnose sJIA sehr hilfreich. Wir richteten unser Hauptaugenmerk auf die S100-Proteine, IL-18 und sIL2-R. IL-1, IL-6, TNF- $\alpha$ brachten für die Diagnosestellung keine zusätzliche Information, rundeten aber die Information über die Inflammation zu verschiedenen Zeitpunkten der Erkrankung ab [3]. Wir beließen bzw. adaptierten die Therapie anhand dieser Werte immer in Zusammenschau mit dem klinischen Bild und den anderen Laborwerten. Über einen Beobachtungszeitraum von
3 Monaten nach Beendigung bzw. Umstellung der Therapie haben die Zytokinprofile bisher unsere Anforderungen erfüllt.

\section{Patientin 1}

Bei der Patientin 1 konnten wir unter laufender Therapie die Diagnose sJIA bestätigen und im Verlauf das Ende der bisher monophasischen Erkrankung feststellen.

\section{Patient 2}

Beim Patienten 2 war der Verlauf der Gelenkserkrankung mit lediglich anfänglichen systemischen Krankheitszeichen unter der Therapie mit Canakinumab unzufriedenstellend. Nach Kenntnis der hohen Calprotectin-Werte mit normalem IL-18 konnten wir die Therapie ohne Angst vor einem Makrophagen-Aktivierungs-Syndrom auf Methotrexat und Etanercept bzw. in der Folge auf Adalimumab verändern.

Eine Uveitis tritt bei einer sJIA nur extrem selten auf [10]. Als Nebenbeobachtung hatte Canakinumab offenbar die Potenz, das Auftreten der schwersten Uveitis über einen Zeitraum von 2 Jahren zu verhindern
[12]. Der Verlauf während unserer Beobachtungszeit lässt an eine JIA, Polyarthritis, ANA positiv, HLA-B27 negativ, CCP-AK schwach positiv, RF negativ, mit Iridozyklitis denken.

\section{Patientin 3}

Die Patientin 3 hatte klinisch eine kurzdauernde bisher monophasische Erkrankung. Da die Patientin während des gesamten Spitalaufenthaltes trotz der Fieberzacken in gutem Allgemeinzustand war und die für die Klassifizierung notwendigen Laborbefunde sehr moderat bzw. nur grenzwertig verändert waren, haben wir die Diagnose MAS, für die die EULAR-Kriterien 2016 [7] erfüllt waren, zunächst negiert. Wir haben sie mit der vorläufigen Diagnose „kurzer monophasischer Schub einer sJIA“ nach Hause entlassen. Die nachträglich eingetroffenen Befunde mit exzessiv erhöhten Zytokinen und S100-Proteinen sowie die genetischen Befunde lassen aber doch an ein (abortives) MAS im Rahmen der heterozygoten HLH-Mutation UNC13D denken. 
Die S100-Proteine und Zytokine sind über einen Zeitraum von mehr als einem Jahr langsam gefallen ohne klinische oder sonstige laborchemische Krankheitszeichen [3]. Den weiteren Verlauf mit möglichen Episoden von sJIA oder Makrophagen-Aktivierungs-Syndrom werden wir kritisch beobachten.

Bei entsprechender Verfügbarkeit erscheint uns das Zytokinprofil mit Calprotectin, S100A12, sIL2-R, IL-18, IL-1, IL-6, TNF- $\alpha$, Interferon-y sinnvoll bei Patienten mit Verdacht auf sJIA bzw. MAS, um rechtzeitig die Diagnose stellen bzw. ausschließen zu können und um innerhalb des „window of opportunity“ mit einer IL-1- oder IL-6-Blockade beginnen zu können und die Therapie nach Abklingen der Entzündungszeichen, der S100-Proteine und Zytokine ohne Gefahr für ein Rezidiv der sJIA oder eines Makrophagen-Aktivierungs-Syndroms beenden zu können.

\section{Interessenkonflikt}

G. A: Kongressunterstützungen, Literaturservice, Vortragshonorare von AbbVie, Actelion, Pfizer, Roche, Sobi, CSL Behring, Shire

H. W.: kein Interessenkonflikt

\section{Einhaltung ethischer Richtlinien}

Der Autor gibt an, dass Einverständniserklärungen der Eltern der Patienten für die Publikation der Fälle vorliegen.
Autoren

Gottfried Artacker ${ }^{1}$
Hermann Wolf $^{2}$
${ }^{1}$ Kinderrheumaambulanz Donauspital/
SMZO Wien;
${ }^{2}$ Immunologische Tagesklinik Wien

\section{Korrespondenzadresse}

\section{Dr. Gottfried Artacker}

Wiener Krankenanstaltenverbund Abteilung für Kinder- und Jugendheilkunde Sozialmedizinisches Zentrum Ost Donauspital

Langobardenstr. 122, 1220 Wien

Österreich

\section{Literatur}

[1] Vastert SJ, de Jager W, Noordman BJ et al. Effectiveness of first-line treatment with recombinant interleukin-1 receptor antagonist in steroid-naive patients with new-onset systemic juvenile idiopathic arthritis: results of a prospective cohort study. Arthritis Rheum 2014; 66 (4): 1034-1043

[2] Hinze $\mathrm{CH}$, Holzinger D, Lainka E et al; PRO-KIND SJIA project collaborators. Practice and consensus-based strategies in diagnosing and managing systemic juvenile idiopathic arthritis in Germany. Pediatric Rheumatology 2018; 16: 7

[3] Feist E, Mitrovic S, Fautrel B. Mechanisms, biomarkers and targets for adult-onset Still`s disease. Nat Rev Rheumatol 2018; 14: 603-618

[4] Xia Y, Cui P, Li Q et al. Extremely elevated IL18 levels may help distinguish systemic-onset juvenile idiopathic arthritis from other febrile diseases. Braz J Med Biol Res 2017; 50(2): e5958

[5] Leek AP, ter Haar NM, Wienke J et al. Interleukin 18 as a diagnostic biomarker in Systemic onset Juvenile Idiopathic Arthritis. University Medical Center Utrecht, Poster Paediatric Rheumatology European Society Congress, Athen/Griechenland, 14.-17. September 2017
[6] Hazen MM, Woodward AL, Hofmann I et al. Mutations of the hemophagocytic lymphohistiocytosis-associated gene UNC13D in a patient with systemic juvenile idiopathic arthritis. Arthritis Rheum 2008; 58(2): 567-570

[7] Ravelli A, Minoia F, Davi S et al. 2016 Classification Criteria for Macrophage Activation Syndrome complicating Systemic Juvenile Idiopathic Arthritis. Ann Rheum Dis 2016; 75: 481-489

[8] Lin M, Park S, Hayden A et al. Clinical utility of soluble interleukin-2 receptor in hemophagocytic syndromes: a systematic scoping review. Ann Hematol 2017; 96: 1241-1251

[9] Henter J-I, Horne A, Arico M et al. HLH-2004: Diagnostic and therapeutic guidelines for hemophagocytic lymphohistiocytosis. Pediatr Blood Cancer2007; 48: 124-131

[10] Heiligenhaus A, Minden K, Föll D, Pleyer U. Uveitis in juvenile idiopathic arthritis. Dtsch Arztebl Int 2015; 112: 92-100

[11] Schmeling H, Horneff G. Etanercept and uveitis in patients with juvenile idiopathic arthritis. Rheumatology 2005; 44 : 1008-1011

[12] Brambilla A, Caputo R, Cimaz R, Simonini G. Canakinumab for Childhood Sight-threatening Refractory Uveitis: A Case Series. J Rheumatol 2016; 43: 1445-1447

[13] Hausmann JS. Targeting cytokines to treat autoinflammatory diseases. Clin Immunol 2018 Oct 28. pii: S1521-6616(18)30432-7.

[14] Shenoi S, Wallace CA. Diagnosis and Treatment of Systemic Juvenile Idiopathic Arthritis. J Pediatr 2016; 177: 19-26

[15] Kawashima M, Yamamura M, Taniai M et al. Levels of interleukin-18 and its binding inhibitors in the blood circulation of patients with adult-onset Still's disease. Arthritis Rheum 2001; 44(3): 550-560

Bibliografie

DOI https://doi.org/10.1055/a-0891-7529

arthritis + rheuma 2019; 39: 196-200

(c) Georg Thieme Verlag KG Stuttgart · New York ISSN 0176-5167 